\title{
Automation of Air Traffic Management Using Fuzzy Logic Algorithm to Integrate Unmanned Aerial Systems into the National Airspace
}

\author{
Kouroush Jenab $^{1}$, Joseph Pineau ${ }^{2}$ \\ ${ }^{1}$ Faculty of Department of Engineering \& Technology Management, Morehead State University, USA \\ ${ }^{2}$ Avionics Engineer, Rockwell Collins, USA
}

\begin{tabular}{l} 
Article Info \\
\hline Article history: \\
Received Nov 1, 2017 \\
Revised Jan 22, 2018 \\
Accepted Jul 26, 2018 \\
\hline
\end{tabular}

Keyword:

Air traffic control

ATM

Fuzzy logic

Next generation air traffic

management

NextGen

Safety

UAS

\begin{abstract}
Unmanned Aircraft Systems (UAS) have been increasing in popularity in personal, commercial, and military applications. The increase of the use of UAS poses a significant risk to general air travel, and will burden an already overburdened Air Traffic Control (ATC) network if the Air Traffic Management (ATM) system does not undergo a revolutionary change. Already there have been many near misses reported in the news with personal hobbyist UAS flying in controlled airspace near airports almost colliding with manned aircraft. The expected increase in the use of UAS over the upcoming years will exacerbate this problem, leading to a catastrophic incident involving substantial damage to property or loss of life. ATC professionals are already overwhelmed with the air traffic that exists today with only manned aircraft. With UAS expected to perform many tasks in the near future, the number of UAS will greatly outnumber the manned aircraft and overwhelm the ATC network in short order to the point where the current system will be rendered extremely dangerous, if not useless. This paper seeks to explore the possibility of using the artificial intelligence concept of fuzzy logic to automate the ATC system in order to handle the increased traffic due to UAS safely and efficiently. Automation would involve an algorithm to perform arbitration between aircraft based on signal input to ATC ground stations from aircraft, as well as signal output from the ATC ground stations to the aircraft. Fuzzy logic would be used to assign weights to the many different variables involved in ATM to find the best solution, which keeps aircraft on schedule while avoiding other aircraft, whether they are manned or unmanned. The fuzzy logic approach would find the weighted values for the available variables by running a simulation of air traffic patterns assigning different weights per simulation run, over many different runs of the simulation, until the best values are found that keep aircraft on schedule and maintain the required separation of aircraft.
\end{abstract}

Copyright (C) 2018 Institute of Advanced Engineering and Science. All rights reserved.

\section{Corresponding Author:}

Kouroush Jenab,

Faculty of Department of Engineering \& Technology Management, Morehead State University, Morehead, KY, USA.

Email: k.jenab@moreheadstate.edu

\section{INTRODUCTION}

Integration of UAS into the National Airspace (NAS) will increase the standard of living for all people by replacing and/or assisting humans in daily tasks. The problem that exists with UAS integration is that the Air Traffic Management (ATM) system is not designed to handle an increase of UAS aircraft on top of commercial transportation and personal aircraft [1]-[32]. ATC personnel are already burdened with the current amount of traffic such that integration of UAS into the NAS is currently not possible [2]. 
The current infrastructure of ATM is based on past technology and needs to be updated to use a different architecture and advanced technology [2]. The US Federal Aviation Administration (FAA) has recognized this shortcoming and has spearheaded investigation into a new architecture, the Next Generation (NextGen) ATM System [3]. The NextGen ATM system is a holistic architectural System of Systems (SoS) approach to integrating many systems into one functional overall system that is greater than the sum of its parts [2]. NextGen will include necessary regulatory guidance, national standards for airworthiness and interface descriptions, technological improvements, public relations, and substantial government investment [4].

In [33], a variety of control systems for a Stop-Rotor Unmanned Aerial Vehicle (UAV) have been developed for hover flight. A mathematical model was presented to captures the stop-rotor dynamics. However, the authors mentioned that the Stop-Rotor UAV was unstable in hover. So, the use of fuzzy mathematical model would help to stabilize the hover dynamics. Compared between performances of current approaches, air traffic management which is a decision making process suffers from uncertainty situations resulted from 1) air traffic controller is not well understood 2) aircraft-to-aircraft conflict decision aid, and 3) inaccurate automation. Therefore, the focus of this paper is on one specific technological improvement, which could handle the increase in number of aircraft, a fuzzy logic algorithm to automate aircraft arbitration safely and efficiently.

\section{ATM AUTOMATION ISSUES}

The issue is that an automated ATM system is not currently in place, nor possible to implement without architectural changes. The architectural changes include all aircraft in the NAS being equipped with:

a. Flight Management System (FMS) capable of Required Navigational Performance (RNP) Area Navigation (RNAV) to provide for accurate positional information and future aircraft positions with high precision

b. Automated Dependent Surveillance Broadcast (ADS-B) in/out to transmit/receive aircraft location, flight plan information, performance characteristics/limitations, and health status; between aircraft and Air Traffic Control (ATC) ground stations, as well as other aircraft.

ATC ground stations would also need to be capable of ADS-B in/out to receive the transmitting aircraft's data and send out commands to the aircraft to modify flight plans as appropriate to keep required separation between aircraft. The ground stations will need to be placed equidistant around the country with a slight overlap in each station's Area of Responsibility (AoR). The overlap in the AoR will allow for one or more ground stations to coordinate the handoff of aircraft moving into or out of their AoR. Figure 1 shown the AoRs for multiple ground stations.

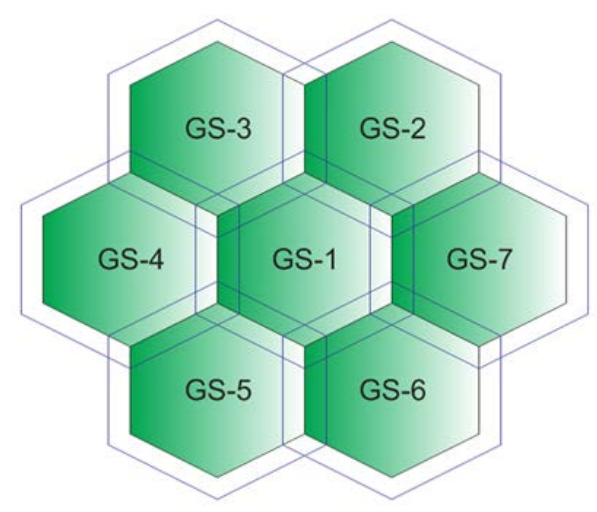

Figure 1. Ground station AoR layout

Each ground station will need to have separate redundant systems for ADS-B communication and fuzzy logic algorithm for system redundancy and error checking [2]. Status of the ground station will need to be shared with adjacent ground stations to allow for shutting down of a malfunctioning ground station AoR and diverting traffic around it. The exchange between ground stations could occur through ADS-B and/or through secure direct Internet connections. The ground stations would have the final say on any modifications of flight plans, while the data link between aircraft would ensure that if a ground station 
becomes inoperative while aircraft are within its AoR, separation criteria between aircraft could still be accomplished by collision avoidance logic embedded within in aircraft. Figure 2 shows the ADS-B communication links between Unmanned Aerial Vehicles (UAVs) and Ground Stations (GS) with and without an overlap of the AoR.

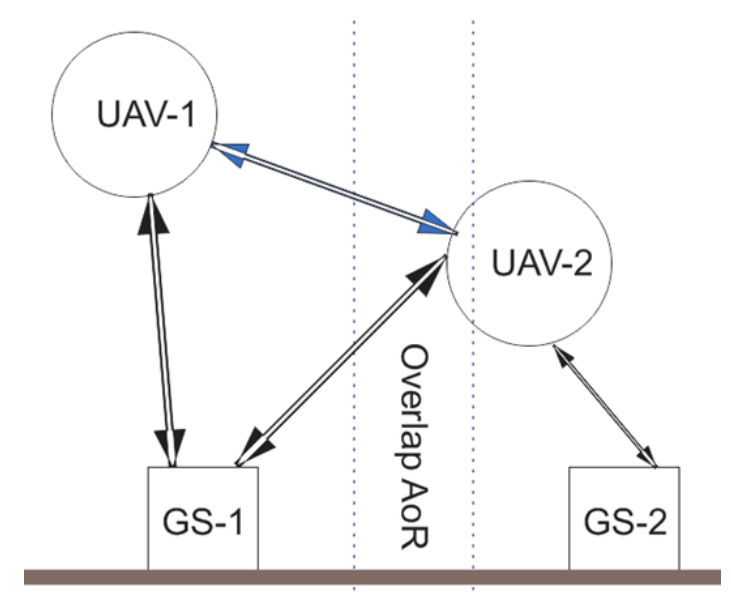

Figure 2. ADS-B communication

Automating the ATM system requires taking humans out of the loop as much as possible [2]. One way to do this is with a fuzzy logic algorithm to take in data from aircraft and adjust their flight plans as needed to ensure required separation. Much work has already been accomplished in fuzzy logic algorithms using sensor and communication information to keep vehicles from colliding with other vehicles or other objects [5]. One algorithm using fuzzy logic will be analyzed in the case study below.

\section{FUZZY LOGIC THEORY}

Fuzzy logic differs from standard logic of 1's and 0's, by introducing a confidence factor to a result. When concepts are vague, such as fast or slow, the confidence factor applies to the result on a scale of $0-1$ on how true the answer is. For example, a question on whether a dog is fast or slow may return an answer of fast with a confidence factor of 0.7 and also an answer of slow with a confidence factor of 0.5 . Since speed of fast or slow is a vague concept, a degree as to how true each answer is comes along with the answer. If one was comparing the dog to a jet aircraft, the dog is slow; while if comparing the dog to a snail, the dog is fast. An important observation is to know that the speed of the dog is known exactly, it is only which category the dog belongs to, fast or slow, that is uncertain [6].

Fuzzy logic excels in control systems, especially control systems that have non-linear solutions or imperfectly known rules. Observing an air traffic controller making complex decisions based on heuristic logic can show an example of imperfectly known rules. An expert air traffic controller would then be an expert to advise fuzzy logic controller developers on potential rule statements which take the form of IFTHEN statements which logical operators of AND, OR, and NOT [6]-[7]. Additional Information on fuzzy logic can be found in 'Fuzzy Sets and Fuzzy Logic: Theory and Applications [8].

\section{AUTOMATION AIR TRAFFIC MODEL CASE STUDY}

John R. Clymer, [7] performed a study where fuzzy logic was used to create control rule sets to ATM of aircraft entering an airport approach corridor. The study was to determine if induced adapted fuzzy rules would perform better than non-adapted fuzzy rules and nonlinear algorithms. The details of the study will be described below.

The study used a simulation of a small airport to create fuzzy rules and modify the fuzzy rules with strength values. The airport simulation is limited to an approach queue with a pattern leading to a runway landing. The simulation also has aircraft queue waiting to takeoff from the same runway. An example of the airport layout is provided in Figure 3. 


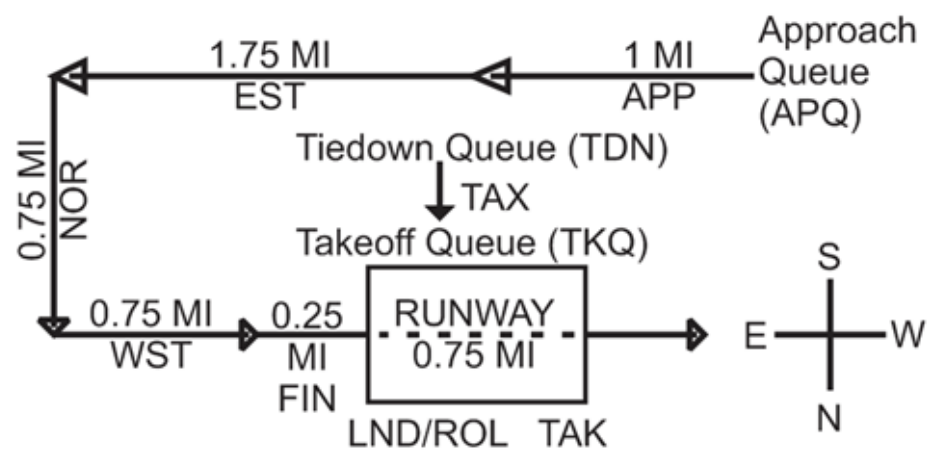

Figure 3. Airport scenario showing an approach pattern starting from a built up queue, with a takeoff queue also in consideration for runway space and time

A discrete symbol key has been provided to assist in understanding the different areas of a normal aircraft pattern shown in the figure above. The discrete symbol key can be found in Table 1.

Table 1. Discrete Symbol Key for Clarification of Figure 1 Discrete Symbols

\begin{tabular}{cc}
\hline Discrete Symbol & Discrete Name \\
\hline APQ & Approach Queue \\
APP & Approach \\
EST & East \\
NOR & North \\
WST & West \\
FIN & Final Approach \\
LND/ROL & Land/Roll \\
TDN & Tie-down Queue \\
TAX & Taxiing \\
TKQ & Takeoff Queue \\
TAK & Takeoff \\
CLR & Clear \\
\hline
\end{tabular}

The fuzzy logic rules were to use the different discrete states of aircraft and their positions and velocities in the Airport Scenario to adjust the wait times to increase airport efficiency and reduce the number or near collisions. An example rule is show:

a. DECISION approach_queue_decision=wait 25

FACT aq_aircraft_ahead_speed=same_speed

FACT aq_aircraft_ahead_position=near

Another example of a rule used in the simulation is one to keep aircraft separation on the runway, this rule is shown below:

b. IF

ANY^ Approach_Aircraft_State=FIN

THEN

Takeoff_Decision=Wait_in_Takeoff_Queue

All fuzzy functions take the form of an S-function where A, B, and C are integer values with both A and $\mathrm{C}$ positive. The $\mathrm{X}$ values of the function once calculated using $\mathrm{A}, \mathrm{B}$, and $\mathrm{C}$; are rounded to the nearest integer value and normalized to a scale of 0 through 100. Table 2 shown S-function variable definitions for graph

Table 2. S-function Variable Definitions for Graph shown in Figure 2

\begin{tabular}{cc}
\hline Variable & Definition \\
\hline A & The spread of the membership function \\
B & Controls the slope of the function \\
C & Kept '0' unless a symmetrical function is desired \\
C + A & Mean Value of the function \\
X & Domain elements of system control \\
\hline
\end{tabular}


The equation of the S-function is shown:

c. (3) $\mathrm{A}(\mathrm{x})=100 /\{1+[(1 / \mathrm{A})(\mathrm{x}-\mathrm{C})] * * \mathrm{~B}\}$

A graph of the S-function is shown in Figure 4.

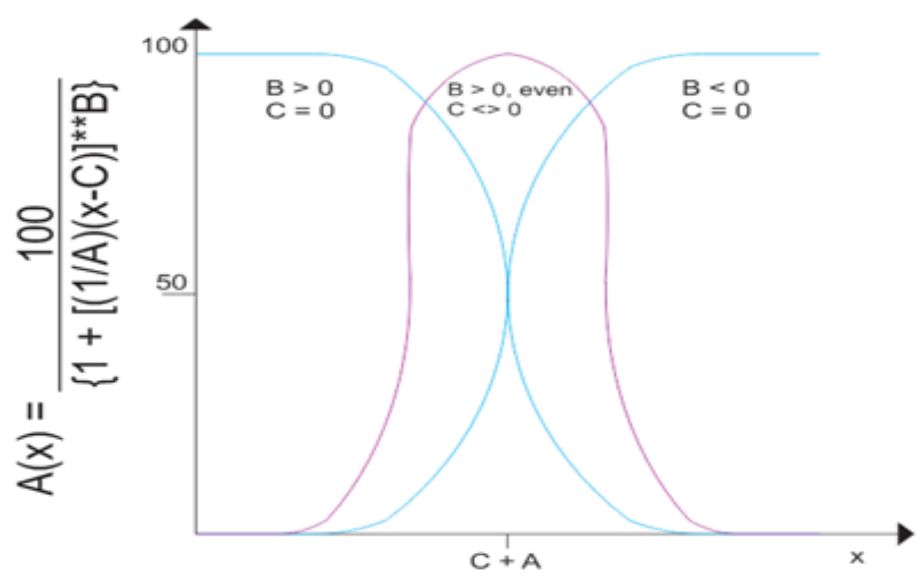

Figure 4. S-Function Graph showing curve variations given differing values of B and C variables

The variables of the S-Function are then added to a user generated rule file of fuzzy facts. An example of some fuzzy facts showing the fuzzy object name, its legal value, A, B, C, and X values is shown :

d. fuzzyfact(aq_aircraft_ahead_position)=close: 2500, 2, 0, slant_range

fuzzyfact(aq_aircraft_ahead_position)=near: 2500, 2, 5000, slant_range

fuzzyfact(aq_aircraft_ahead_position)=far: 7500, 13, 0, slant range

fuzzyfact(aq_aircraft_ahead_speed)=not_closing_slow: 5, 2, 0, closing_velocity

fuzzyfact(aq_aircraft_ahead_speed)=same_speed: 5, 2, 9, closing_velocity

fuzzyfact(aq_aircraft_ahead_speed)=closing_slow: 5, 2, 17, closing_velocity

fuzzyfact(aq_aircraft_ahead_speed)=closing_fast: 5, 2, 25, closing_velocity

fuzzyfact(aq_aircraft_ahead_speed)=closing_very_fast: 28, -13, 0, closing_velocity

Fuzzy facts make up different sets of cases, and one or more cases make up an overall concept. If one case is shared by several concepts then the decision is ambiguous. This would also mean that the rules, which make up the cases, are involved in multiple concepts. This idea is what makes the rules 'fuzzy'. The ambiguity, which results, can then be resolved by comparing the strength of each concept. An algorithm called 'bucket brigade method' was used to modify the strength of the fuzzy facts. The modification would determine a support value based on the sum of bids for all rules concluding the fact, and normalize that number to an integer between 0 and 100. This normalized number is then called the Confidence Factor (CF).

The study determines bids in the following manor: "A rule bid is the product of the rule support, proportional to specificity and rule strength, divided by eight hundred. The rule support is the sum of the support values of all facts or composite facts comprising the rule premise" [7]. "The support value of a composite fact is the average support value of the facts comprising it. Rule strength is the confidence value associated with the conclusion being pursued (integer 0 to 100). Specificity is the number of facts plus composite facts in the premise. A specific rule has a higher bid than a more general rule. A bid is not made unless all facts and composite facts in the premise are evaluated true. When a bid is made, it is randomized using a multiplier (1+0.15*Random) to enhance convergence to optimal system operation” [7].

Ambiguity is determined based on the CF of each possible decision. If one decision has a high CF while other possibilities have a low CF, then there is low ambiguity. If the average difference between possible decisions is small, then the ambiguity is high. Modification of rule strengths is performed on only the rules that support the decision. Subtracting the rule bid from its strength and using the bid of each rule to add to the supporting rules' strength does this. As the simulation is run, rule strengths are modified either up or down until the rules reach a steady state where the optimum solution resides for a given rule set.

An example is given of one area of the overall program. In the example the program is determining the appropriate timing for aircraft in the Approach Queue. The idea is to keep the aircraft from beginning their approach until sufficient time between the aircraft ahead in the approach is realized. The gap in time 
between aircraft on approach, will allow an aircraft on the ground to takeoff in between each aircraft that lands. Example rules for this process are shown on the following page.

e. RULE001:

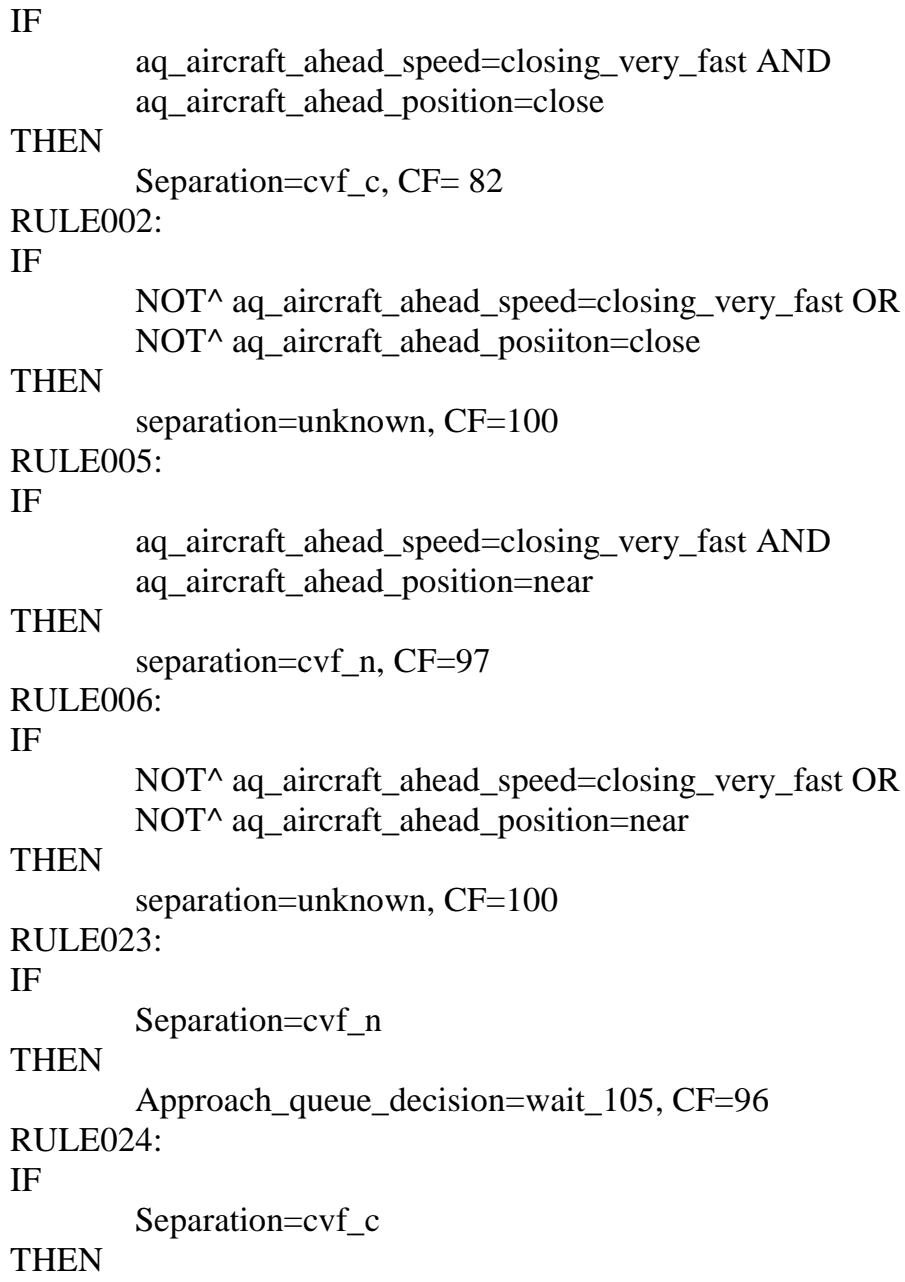

Approach_queue_decision=wait_105, CF 78

When one decision has a much greater $\overline{C F}$ than any other possibility, then it is the decision to be used to control the aircraft wait time. However, when ambiguity is high there is not one decision with a much greater CF than any other and a separate method is used. Figure 5 shown understanding of the method.

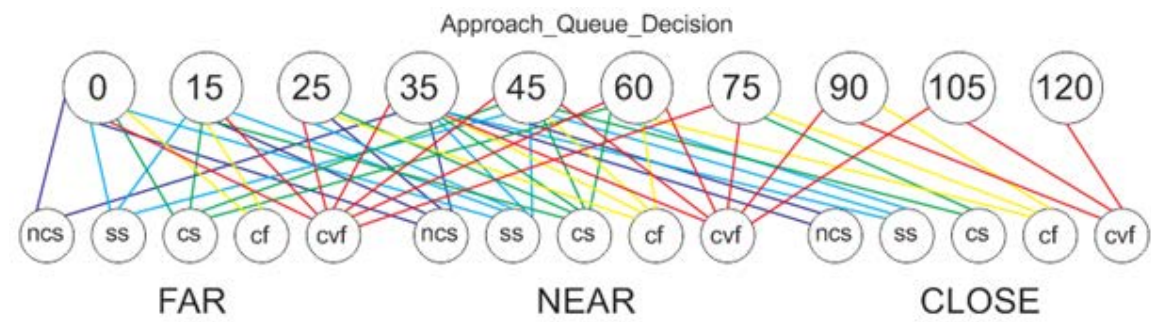

Figure 5. Fuzzy rule induced values correlating to fuzzy values of far, near, and close; and their associated approach queue decision values for wait time

Assuming that 60 seconds has the highest CF, and is therefore the best value; but 25, 35, and 45 seconds have CF comparable to that of the 60 seconds decision. In this situation the average ordinal value of the possible decisions is determined, and the difference between this average ordinal value and the best decision ordinal value results in a delta ordinal value. The delta ordinal value is then multiplied by 5 and is called the adjustment. Then the wait time can be computed as $60+$ adjustment. 
f. Average Ordinal Value $=[(3+4+5+6) / 4]=1.5$

Delta Ordinal Value $=(1.5-6)=-1.5$

Adjustment Value $=(-1.5 * 5)=-7.5$

Wait Time $=[(60+(-7.5)]=52.5$

Using this method allows for a continuous range to be used for aircraft wait time to keep separation, from the discrete fuzzy logic sets and rules. The same method is used for the other scenarios in this airport approach simulation using these and other fuzzy rule sets. The results of the experiment with fuzzy logic to control aircraft in a simulated aircraft environment showed that the adapted fuzzy rules were superior to both, un-adapted fuzzy rules, and a conventional control algorithm. Table 3 and Figure 6 show the results of the three control rules when comparing Approach Queue wait time, and Potential Collisions.

Table 3. Performance Comparison Variable Definitions Defined for the Legend that is Shown in Figure 6

\begin{tabular}{|c|c|}
\hline $\begin{array}{l}\text { Variable } \\
\end{array}$ & Definition \\
\hline $\begin{array}{l}\text { ALG } \\
\text { APQ }\end{array}$ & Standard Algorithm Approach Queue Wait Time \\
\hline ALG & Standard Algorithm Potential Collisions \\
\hline $\begin{array}{l}\text { NOM } \\
\text { APQ }\end{array}$ & $\begin{array}{c}\text { Un-adapted Fuzzy Rules Approach Queue Wait } \\
\text { Time }\end{array}$ \\
\hline $\begin{array}{l}\text { NOM } \\
\text { COL }\end{array}$ & Un-adapted Fuzzy Rules Potential Collisions \\
\hline BST APQ & $\begin{array}{l}\text { Adapted Fuzzy Rules Approach Queue Wait } \\
\text { Time }\end{array}$ \\
\hline BST COL & Adapted Fuzzy Rules Potential Collisions \\
\hline
\end{tabular}

\section{AIRPORT PERFORMANCE COMPARISON}

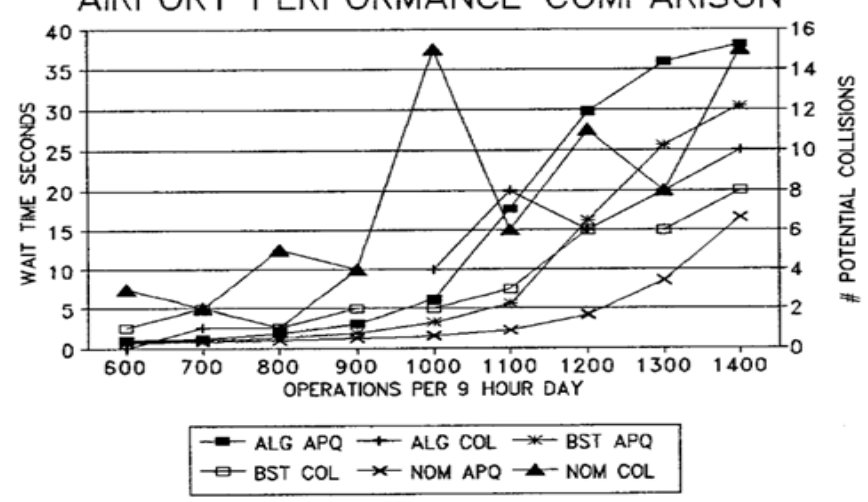

Figure 6. performance comparison. reprinted from “induction of fuzzy rules for air traffic control," by r. j. clymer, 1995, systems, man and cybernetics, intelligent systems for the 21st century, 2, p.7. copyright 1995 by IEEE

\section{AUTOMATION ALGORITHM USING FUZZY LOGIC}

A fuzzy logic algorithm is suggested which will take in ADS-B data from each UAS in the AoR of each ground station. The algorithm will analyze the collected data to search for possible future collision paths between two or more UAS. If a collision path is found, then the algorithm will determine the best solution of modification to one or more UAS's flight plans to avoid a collision or near miss. ADS-B data to be analyzed from each UAS are listed:

a. UAS Aircraft Type

The UAS Aircraft Type will be used to access a database with the performance characteristics and limitations of each aircraft

b. UAS Health Status

The UAS Health Status will be used for priority determination and emergency procedures

c. UAS RNP Status

UAS RNP Status will be used to determine separation requirements based on RAIM and Predictive RAIM values 
d. UAS Present Position

UAS Present Position will be used to know the Position of the UAS when the message was sent

e. UAS Ground Track

UAS Ground Track will be used to know the current path over the ground of the UAS

f. UAS Ground Speed

g. UAS Altitude

UAS Ground Speed will be used to know the current ground speed of the UAS

h. UAS Flight Plan

UAS Altitude will be used to know the altitude of the UAS

UAS Flight Plan will be used to know the future Position, Ground Track, Ground Speed, and Altitude of the UAS

i. UAS Schedule (Time to go, Time on target)

UAS Schedule will be used in the priority calculation to determine which aircraft's flight plan will be modified, how much it will be modified, and in what way (Speed, Course, Altitude)

j. UAS Message Sent Time

UAS Message Sent Time will be used to predict where the UAS is after the ground station has received the message to account for latency. The information is then parsed by the fuzzy logic system and fed into the existing fuzzy rules/facts. Some example rules/facts are listed:

k. If UAS is traveling East (or 360 degrees) Then Altitude Band=150, 250, 350

1. If UAS is traveling West (or 180 degrees) Then Altitude Band=100, 200, 300

m. If UAS datalink is lost AND traveling East (or 360 degrees) Then Altitude Band=450

n. If UAS datalink is lost AND traveling West (or 180 degreees) Then Altitude Band=400

o. If UAS Health is NOT Good Then Altitude Band=500

p. If UAS Altitude is less than Digital Aeronautical Flight Information File (DAFIF) database Then Altitude Band=Higher Band

q. If Future Position is Close to other UAS Future Position Then Modify Flight Plan UAS Determination=True

r. If Future Position is Close to other UAS Future Position Then Collision Path=True

s. If Future Position is within GeoFence Then Modify Flight Plan UAS=True

t. If Potential Collision in less than 30 seconds then Collision Time=Now

u. If Potential Collision in less than 2 minutes then Collision Time=Soon

v. If Potential Collision in less than 5 minutes then Collision Time=Later

w. If Potential Collision in 5 minutes or more then Collision Time=Eventually

$\mathrm{x}$. If UAS RNP status is Good then Collision area=Small

y. If UAS RNP status is Degraded then Collision area=Medium

z. If UAS RNP status is Failed then Collision area=Large

The above rules/facts are simplified to try to show the possible relationships. An example of what a rule may actually look like is shown below:

IF E_UAS.Altitude Band=250 AND Future_Positions (in AoR) do NOT pass through GeoFence AND Health_Status=Good AND Schedule is=On_Time AND Collision_Path=TRUE AND Collision_Time=SOON AND Aircraft_Type1=0x123 AND Aircraft_Type2=0x456 THEN Modify Aircraft_Type2.Flight_Plan.Current_Velocity $=50$ knots for 45 seconds AND Modify Aircraft_Type2.Ground_Track=085 degrees

\section{CONCLUSION}

The recommendation of this paper is to pursue a fuzzy logic solution to integrate UAS into the NAS. Fuzzy fact and Fuzzy rule development should be perused using expert Air Traffic Control personnel as consultants providing heuristic knowledge of safe ATM operations. The fuzzy facts and rules developed should be used in an air traffic simulation to adapt the weights of the fuzzy facts and rules until a stable system has emerged. Testing of the resulting stable system in the real world should then be conducted to refine the fuzzy system further in controlled restricted airspace. This testing should also include stress testing with swarms of UAS, as well as emergency conditions and procedures. Implementation of the NextGen ATM system using fuzzy logic control should then be phased in over time starting with more rural locations and expanding over time to one day include controlling previously conventionally controlled cargo and passenger aircraft.

Dependencies to an automated ATM system are that all aircraft must be equipped with the FAA regulated minimum required avionics to meet all regulations including 'sense and avoid'. Other dependencies include the development and testing of two separate fuzzy logic methods to optimize automated control of 
UAS and keep required separation in a redundant and self-analyzing/correcting manor. Research into electromagnetic bandwidth allocation and division would increase the amount of data each UAS can send over a datalink and should be pursued. Data-link security and encryption also needs to be pursued to prevent unintentional or malicious signal loss or corruption. Development of low cost and low weight sensors for obstacle detection and collision avoidance will also be important. Environmental hazards associated with transmission and reception of data-link data need to also be addressed and mitigated. Other future research can look into the possibility of super or quantum computers in processing data of the fuzzy logic control system to find a more perfect navigational solution.

\section{REFERENCES}

[1] Kopardekar, P. (2014). Unmanned Aerial System (UAS) Traffic Management (UTM): Enabling Low-Altitude Airspace and UAS Operations.

[2] Rediess, H. (2004, October). Infrastructure concept for automated aircraft and air traffic operations. The 23rd IEEE Digital Avionics Systems Conference DASC 04. 1(1-C), 11-12.

[3] McCallie, D., Butts, J., \& Mills, R. (2011). Security analysis of the ADS-B implementation in the next generation air transportation system. International Journal of Critical Infrastructure Protection, 4(2), 78-87.

[4] Nextgen Priorities. (2015, October). Joint Implementation Plan. FAA. Retrieved on April 2016 from http://www.faa.gov/nextgen/media/ng_priorities.pdf.

[5] Degtyarev, O. V., \& Zubkova, I. F. (2012). Methods and features of mathematical simulation of air traffic management systems. Journal of Computer and Systems Sciences International, 51(4), 535-548. doi:10.1134/s1064230712030045

[6] What is 'fuzzy logic'? Are there computers that are inherently fuzzy and do not apply the usual binary logic?. (1999, October). Scientific American.

[7] Clymer, J. R. (1995, October). Introduction of fuzzy rules for air traffic control. IEEE International Conference on Systems, Man and Cybernetics, 1995. Intelligent Systems for the 21st Century, 2(1), 1495-1502. doi: $\{10.1109 /$ icsmc.1995.537984

[8] Clothier, R. A., Fulton, N. L., \& Walker, R. A. (2008). Pilotless aircraft: the horseless carriage of the twenty-first century?. Journal of Risk Research, 11(8), 999-1023. doi: 10.1080/13669870802323353

[9] Autry, T., \& Andersen, A. (2016). Supercomputers, super efficiency. ASHRAE Journal, 58(1), 38-39.

[10] Baraldi Sesso, D., Vismari, L. F., \& Camargo, J. B. (2014, May). An approach to assess the safety of ADS-B based unmanned aerial systems. 2014 IEEE International Conference on Unmanned Aircraft Systems (ICUAS), 669-676. doi: 10.1109/icuas.2014.6842311

[11] Bedford, M. A. (2013). Unmanned Aircraft System (UAS) Service Demand, 2015-2035. Retrieved on May 2016 from https://fas.org/irp/program/collect/service.pdf.

[12] Bearne, S. (2015). New rules threaten to derail amazon drone delivery plans. Retail Week. Retrieved on May 2016 from https://www.retail-week.com/sectors/general-merchandise/new-rules-threaten-to-derail-amazon-dronedelivery-plans/5072114.fullarticle

[13] Cummings, M. L., \& Tsonis, C. (2005, September). Deconstructing complexity in air traffic control. Proceedings of the Human Factors and Ergonomics Society Annual Meeting, 49(1), 25-29. doi:10.1177/154193120504900107

[14] D'Andrea, R. (2014). Guest Editorial Can Drones Deliver? Automation Science and Engineering, IEEE Transactions on, 11(3), 647-648. doi:10.1109/tase.2014.2326952

[15] Etherington, D. (2015). Amazon's delivery drones could find you wherever you are. New York: AOL Inc.

[16] Klir G.J \& Bo, Y. (1995). Fuzzy sets and fuzzy logic, theory and applications. Prentice-Hall Inc., NJ, USA.

[17] Gimenes, R. A. V., Vismari, L. F., Avelino, V. F., Camargo Jr, J. B., de Almeida Jr, Jorge R, \& Cugnasca, P. S. (2014). Guidelines for the integration of autonomous UAS into the global ATM. Journal of Intelligent \& Robotic Systems, 74(1), 465-478. doi:10.1007/s10846-013-9945-0

[18] Ison, D. C., Terwilliger, B., \& Vincenzi, D. (2014). Privacy, restriction, and regulation involving federal, state and local legislation: More hurdles for unmanned aerial systems (UAS) integration? Journal of Aviation/Aerospace Education \& Research, 24(1), 41. doi:10.15394/jaaer.2014.1606

[19] Maroney, D., Bolling, R., Heffron, M., \& Flathers, G. (2009). Evaluating sensor technology for UAS collision avoidance. IEEE Aerospace and Electronic Systems Magazine, 24(4), 36-43. doi:10.1109/MAES.2009.4839275

[20] Martel, F., Schultz, R., Wang, Z., \& Semke, W. (2011) Flight testing of an ADS-B-based miniature 4D sense and avoid system for small UAS. Infotech @ Aerospace, 29-31. doi:10.2514/6.2011-1419

[21] McAree, O., \& Chen, W. (2013). Artificial situation awareness for increased autonomy of unmanned aerial systems in the terminal area. Journal of Intelligent \& Robotic Systems, 70(1), 545-555. doi:10.1007/s10846-012-9738-x

[22] Paczan, N. M. (2012, October). Integrating UAS into NextGen automation systems. 2012 IEEE/AIAA 31st Digital Avionics Systems Conference (DASC). Retrieved on May 2016 from https://www.mitre.org/sites/default/files/pdf/12_3347.pdf

[23] Risen, T. (2013, 12). Amazon prepares for package delivery via drone. U.S.News \& World Report. Retrieved on May 2016 from http://www.usnews.com/news/articles/2013/12/02/amazon-prepares-for-package-delivery-viadrone

[24] Sachdev, A. (2015). Technology: Unmanned aerial systems in civil airspace. New Delhi: Athena Information Solutions Pvt. Ltd. 
[25] Smith, A. L., \& Air Force Inst. of Tech. Wright-Patterson AFB OH Graduate School of Engineering and Management (2009). UAS collision avoidance algorithm that minimizes the impact on route surveillance. Retrieved on May 2016 from http://www.dtic.mil/dtic/tr/fulltext/u2/a496726.pdf

[26] Smith, A. L., \& Harmon, F. G. (2011). UAS collision avoidance algorithm based on an aggregate collision cone approach. Journal of Aerospace Engineering, 24(4), 463-477. doi:10.1061/(ASCE)AS.1943-5525.0000

[27] Sutterfield, K. (2007). ADS-B can expand UAV flights. Aviation Week \& Space Technology, 167(22), 10-11.

[28] Valovage, E. (2006, October). Enhanced ads-b research. 25th IEEE Digital Avionics Systems Conference, 2006 IEEE/AIAA, 1-7. doi:10.1109/dasc.2006.313672

[29] Wang, L., \& Yen, J. (1999). Extracting fuzzy rules for system modeling using a hybrid of genetic algorithms and kalman filter. Fuzzy Sets and Systems, 101(3), 353-362. doi:10.1016/S0165-0114(97)00098-5

[30] Wargo, C. A., Church, G. C., Glaneueski, J., \& Strout, M. (2014). Unmanned aircraft systems (UAS) research and future analysis. Paper presented at the IEEE Aerospace Conference 1-16. IEDD. doi:10.1109/AERO.2014.6836448

[31] Yang, C., Wen, M., Chen, Y., \& Kang, S. (2015). An optimized unmanned aerial system for bridge inspection. Retrieved on April 2016 from http://www.iaarc.org/publications/fulltext/FFACE-ISARC15-2999884.pdf

[32] Zektser, A., \& Griffith, D. (2015). The regulator's perspective: Integrating UAS into the national airspace system. The Air and Space Lawyer, 28(2), 3-4.

[33] Vargas-Clara, A., \& Redkar, S. (2012). Dynamics and control of a stop rotor unmanned aerial vehicle. International Journal of Electrical and Computer Engineering, 2(5), 597-608. doi: http://dx.doi.org/10.11591/ijece.v2i5.1589

\section{BIOGRAPHIES OF AUTHORS}

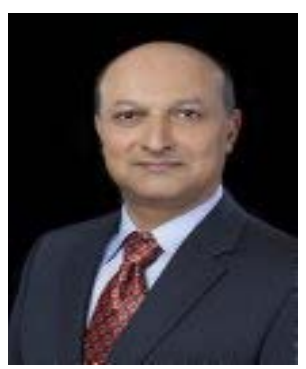

Dr. Kouroush Jenab received his BSc degree from the IE Department at the Isfahan University of Technology (1989), an MSc degree from the IE Department at Tehran Polytechnic (1992), and a PhD degree from the Department of Mechanical Engineering at the University of Ottawa (2005). He served as a Senior Engineer/Manager in auto, and high-tech industries for 20 years. He joined National Research Council Canada as a Research Officer. Currently, he is an assistant professor in Department of Engineering \& Technology Management at Morehead State University. He has published over 170 papers in international journals and conferences based on his experiences in industries.

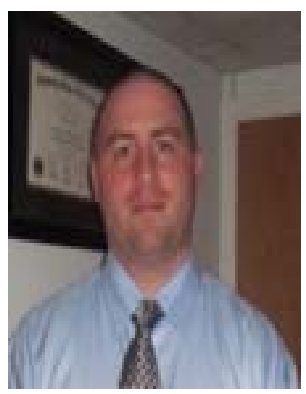

Joseph Pineau received the B.S.E.E degree from the Department of Electrical and Computer Engineering at Manhattan College (2011). He served in the Aerospace industry, helicopters specifically, in varying capacities for 12 years. He served in the US Air Force as an Avionics Technician for 6 years. In 2006, he began working for Sikorsky Aircraft as a test laboratory technician, and then Electrical Integration and Test Engineer, and an Avionics Test Engineer. Currently, Joseph Pineau is a Platform Systems Engineer for Rockwell Collins for the UH-60 helicopter platform. He holds an FAA Airframe Certification, FCC General Radio Telephone Operators License (GRTOL) with Radar Endorsement, and has completed 50 hours of flight training in a Schweizer S300C and S300Cbi helicopter. 